\title{
Semi-Mechanistic Pharmacokinetic Modeling of Lipid Core Nanocapsules: Understanding Quetiapine Plasma and Brain Disposition in a Neurodevelopmental Animal Model of Schizophrenia ${ }^{\mathbb{S}}$
}

\author{
Fernando Carreño, Victória Eteges Helfer, Keli Jaqueline Staudt, Laura Bem Olivo, \\ Karina Paese, Fabíola Schons Meyer, Ana Paula Herrmann, Sílvia Stanisçuaski Guterres, \\ Stela Mari Kuze Rates, (1) Iñaki Trocóniz, and (1)Teresa Dalla Costa \\ Programa de Pós-Graduação em Ciências Farmacêuticas, Faculdade de Farmácia (F.C., V.E.H., K.J.S., L.B.O., K.P., S.S.G., \\ S.M.K.R., T.D.C.), Centro de Reprodução e Experimentação de Animais de Laboratório, Instituto de Ciências Básicas da Saúde \\ (F.S.M.), and Programa de Pós-Graduação em Farmacologia e Terapêutica, Instituto de Ciências Básicas da Saúde (A.P.H.), \\ Universidade Federal do Rio Grande do Sul, Porto Alegre, Rio Grande do Sul, Brazil; Pharmacometrics and Systems \\ Pharmacology Research Unit, Department of Pharmaceutical Technology and Chemistry, School of Pharmacy and Nutrition, \\ University of Navarra, Pamplona, Spain (I.T.); and IdiSNA, Navarra Institute for Health Research, Pamplona, Spain (I.T.)
}

Received May 15, 2020; accepted July 14, 2020

\section{ABSTRACT}

This study investigated plasma and brain disposition of quetiapine lipid core nanocapsules (QLNC) in naive and schizophrenic (SCZ-like) rats and developed a semimechanistic model to describe changes in both compartments following administration of the drug in solution (FQ) or nanoencapsulated. QLNC $(1 \mathrm{mg} / \mathrm{ml})$ presented $166 \pm 39 \mathrm{~nm}$, low polydispersity, and high encapsulation (93.0\% \pm $1.4 \%)$. A model was built using experimental data from total and unbound plasma and unbound brain concentrations obtained by microdialysis after administration of single intravenous bolus dose of FQ or QLNC to naive and SCZ-like rats. A twocompartment model was identifiable both in blood and in brain with a bidirectional drug transport across the blood-brain barrier $\left(\mathrm{CL}_{\text {in }}\right.$ and $\left.\mathrm{CL}_{\text {out }}\right)$. SCZ-like rats' significant decrease in brain exposure with $F Q$ (decrease in $\mathrm{CL}_{\mathrm{in}}$ ) was reverted by
QLNC, showing that nanocarriers govern quetiapine tissue distribution. Model simulations allowed exploring the potential of LNC for brain delivery.

\section{SIGNIFICANCE STATEMENT}

A population approach was used to simultaneously model total and unbound plasma and unbound brain quetiapine concentrations allowing for quantification of the rate and extent of the drug's brain distribution following administration of both free drug in solution or as nanoformulation to naive and SCZ-like rats. The model-based approach is useful to better understand the possibilities and limitations of this nanoformulation for drug delivering to the brain, opening the opportunity to use this approach to improve SCZ-treatment-limited response rates.

\section{Introduction}

The development of new effective therapies for central nervous system (CNS) disorders is a huge challenge for the

This work was supported by the Conselho Nacional de Desenvolvimento Científico e Tecnológico (CNPq/Brazil) [Grant 421767/2016-2] and Coordenação de Aperfeiçoamento de Pessoal de Nível Superior Brazil (CAPES) [Finance Code 001]

The authors declare no conflict of interest.

https://doi.org/10.1124/jpet.120.000109.

S This article has supplemental material available at jpet.aspetjournals.org. pharmaceutical industry (Lindqvist et al., 2013). One important obstacle is the blood-brain barrier (BBB) that significantly limits the ability of therapeutic agents to reach efficacious concentrations at target sites in CNS because of restricted paracellular and transcellular transport (Carreño et al., 2016b). The high variability in the response to treatment observed in chronic schizophrenia (SCZ) may be partially related to BBB dysfunction caused by the disease and consequent alterations on antipsychotic drug transport to CNS (de Klerk et al., 2010).

Antipsychotic drugs are the mainstay of SCZ and schizoaffective disorder pharmacological treatment, and quetiapine

ABBREVIATIONS: ACF, artificial cerebrospinal fluid; APD, antipsychotic drugs; BBB, blood-brain barrier; CL, clearance; CNS, central nervous system; $\mathrm{C}_{\mathrm{u}, \mathrm{B}}$, concentration of quetiapine in brain; $\mathrm{C}_{\mathrm{u}, \mathrm{p}}$, concentration of quetiapine in plasma; $\mathrm{FQ}$, quetiapine solution; $\mathrm{F}_{\mathrm{core}}$, fraction of the

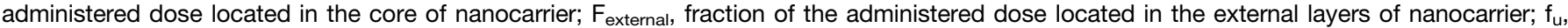
unbound fraction; $F_{\text {surface, }}$, fraction of the administered dose nonencapsulated; HIP, hippocampus; IIV, interindividual variability; $k_{\text {external }}$, first order rate constant describing the release of QTP from the external layers of the nanocarrier; LNC, lipid core nanocapsule; mPFC, medium prefrontal cortex; P-gp, P-glycoprotein; PK, pharmacokinetic; PND, postnatal day; poly(i:c), polyinosinic-polycytidilic acid; PPI, prepulse inhibition; QLNC, quetiapine lipid core nanocapsule; QTP, quetiapine; RSE, relative S.E.; SCZ, schizophrenia; V1, apparent volume of distribution of the central compartment; $\mathrm{V}_{\mathrm{u}, \mathrm{Brain}}$, brain volume of distribution for unbound QTP. 
(QTP), a second-generation antipsychotic drug, has an atypical profile against the positive, negative, and cognitive symptoms of SCZ with a low propensity to induce extrapyramidal side effects (Pira et al., 2004; Yamamura et al., 2009).

Assuming that limited drug delivery to CNS in patients with schizophrenia could be associated with pharmacotherapy resistance, we have developed QTP lipid core nanocapsules (QLNC) aiming to improve drug targeting to the brain (Carreño et al., 2015, 2016a). Indeed it has been shown that nanoparticles are versatile platforms for modifying pharmacokinetic profile and pharmacological performance of loaded drugs (Yuan et al., 2019) and have been used as carriers for drug delivery to the brain (Carreño et al., 2015, 2016b; Dimer et al., 2015; He et al., 2018).

In previous reports, we have shown that QLNC administered intravenously to healthy rats significantly increased total QTP brain exposure, determined in tissue homogenate, in comparison with rats that received nonencapsulated drug (Carreño et al., 2016b). It was also shown that LNC can carry QTP across the BBB, avoiding influx transporters, instead of altering the barrier permeability (Carreño et al., 2016a).

Those studies provided strong evidence that the developed QLNC modulate QTP pharmacokinetics and transport across the $\mathrm{BBB}$, improving drug delivery to the brain. However, the studies were conducted in naive animals, and the influence of SCZ alterations on BBB was not addressed.

Using a neurodevelopmental model of SCZ obtained by administering viral mimic polyinosinic-polycytidilic acid [poly(i:c)] to pregnant rats, we showed that the administration of QLNC, but not QTP, significantly improved the impaired sensorimotor condition, indicating that LNC enhances QTP antipsychotics effect (Carreño et al., 2020).

Understanding pharmacokinetics (PK) of drugs administered in nanoparticles is challenging because just after dosing, two forms of the active component cohabit, one still at the nanoparticle either attached at the surface or inside the particle, and the other already released from the nanodevice, each of them showing their own disposition properties. Not surprisingly, in most of the cases, PK results were obtained from data that do not distinguish between released and encapsulated drug concentrations. Only very few works have attempted to describe the fate these type of pharmaceuticals form by developing mechanistic pharmacokinetic models (Li et al., 2010, 2017; Gilkey et al., 2015; Lindqvist et al., 2016).

The aim of the current investigation is to mechanistically characterize QTP distribution to the brain in an animal model of schizophrenia, providing understanding of the role of the delivery system in CNS diseases in which brain drug disposition might be compromised. To achieve such an objective, data consisting in unbound and total QTP (bound to protein, encapsulated, and unbound), measured in plasma and brain after single intravenous administration of QTP in solution (FQ) or in nanocapsules (QLN) to either naive or SCZ-like animals, were integrated to develop a population PK model.

\section{Material and Methods}

\section{Chemicals and Reagents}

Quetiapine hemifumarate (purity) $\geq 98.0 \%$ was kindly donated by Pratti-Donaduzzi Medicamentos Genéricos (Brazil). Polyinosic-polycytidylic acid sodium salt (P1530) was obtained from Sigma-Aldrich (Brazil).
Polysorbate-80 (Tween 80) was purchased from Delaware Química (Brazil). HPLC ${ }^{\mathrm{a}}$-grade methanol and acetonitrile were obtained from Merck (Brazil), and all other chemicals and reagents used were of analytical grade. Artificial cerebrospinal fluid (ACF) solution consisted of $\mathrm{NaCl} 145 \mathrm{mM}, \mathrm{KCl} 2.7 \mathrm{mM}, \mathrm{CaCl}_{2} 1.2 \mathrm{mM}$, and $\mathrm{MgCl}_{2} 1 \mathrm{mM}$, pH 6.2 (Carreño et al., 2016b).

\section{Quetiapine Lipid Core Nanocapsule Preparation}

QTP lipid-core nanocapsules $(1 \mathrm{mg} / \mathrm{ml})$ were obtained by nanoprecipitation of the preformed polymer as previously published (Carreño et al., 2015). All batches used in this study $(n=5)$ were physicochemically characterized and presented an average size of $166 \pm 39 \mathrm{~nm}$, low polydispersity index $(<0.15)$, and high encapsulation efficiency $(93.0 \% \pm 1.4 \%)$, similar to those LNC data previously published (Carreño et al., 2015, 2016a,b).

A control formulation (FQ, $5 \mathrm{mg} / \mathrm{ml}$ ) was prepared by first dissolving QTP in acetic acid (99\%); then, saline $0.9 \%$ containing polysorbate 80 $(78 \mathrm{mg} / \mathrm{ml}$ ) was added, and the $\mathrm{pH}$ was adjusted to $6.5 \pm 0.5 \mathrm{using}$ $\mathrm{NaOH}(8 \mathrm{M})$.

\section{Pharmacokinetic Study Design}

Animals. This study was approved by the Ethics Committee in Animal Use from the Federal University of Rio Grande do Sul (UFRGS/CEUA \#31001). Male (300-350 g) and female (200-270 g, nulliparous) Wistar breeders were obtained from the Central Animal Facilities of the University's Biochemistry Department (Porto Alegre, Brazil) and housed (three per cage) in a controlled environment (22 \pm $2^{\circ} \mathrm{C}, 65 \%$ humidity in a 12 -hour light/dark cycle) with free access to standard rodent chow and filtered water. The offspring were maintained in the same conditions.

Prenatal poly(i:c) Challenge. Poly(i:c) $4 \mathrm{mg} / \mathrm{ml}$ stock solution was prepared in sterile pyrogen-free $0.9 \% \mathrm{NaCl}$ according to manufacturer instructions, diluted to a final $2-\mathrm{mg} / \mathrm{ml}$ sterile solution in the same vehicle, and stored at $-20 \pm 1^{\circ} \mathrm{C}$ until use.

For the prenatal poly(i:c) challenge, female rats were mated according to a timed-mating breeding scheme (Pritchett-Corning et al., 2009). On the morning of gestational day 15, pregnant damns $(n=6)$ were given a single intravenous bolus injection of $4 \mathrm{mg} / \mathrm{kg}$ of poly(i:c) via the lateral tail vein, and females were housed individually until the birth of their offspring.

On postnatal day (PND) 1, pups were sexed, weighed, and culled to eight pups per litter considering four females and four males (when possible). Weaning occurred at PND21 when pups where housed (four animals per cage) according to sex and litter as previously published (Carreño et al., 2020).

Prepulse Inhibition of Acoustic Startle Reflex. Sensory gating deficits as reflected in impaired prepulse inhibition (PPI) constitute one of the core features of schizophrenia. To confirm that the neurodevelopmental model of schizophrenia described in the literature was adequately induced in our laboratory, SCZ-like deficit in the adult offspring (PND75) was accessed by PPI of the startle response in comparison with a naive rat's offspring according to previously published protocol (Carreño et al., 2020).

Briefly, acclimatization (5 minutes, background noise, $68 \mathrm{~dB}$ ) in the startle chamber (Insight) was followed by 10 initial startle stimuli $(120 \mathrm{~dB})$. The test was started with different trial types in random order: pulse alone $(120 \mathrm{~dB})$, background noise $(68 \mathrm{~dB})$, or prepulse (PP 71, 77, or $85 \mathrm{~dB})+$ pulse. A total of 10 presentations of each trial type were given with an interstimulus interval randomized at $15 \pm 8$ seconds. These results were used during model development.

Surgical Procedure. Forty eight hours before plasma pharmacokinetic and brain microdialysis experiments, adult male and female SCZ-like rats (PND75-85) were anesthetized (ketamine/ xilazine/acepromazine: $100 \mathrm{mg} / \mathrm{kg}, 10 \mathrm{mg} / \mathrm{kg}, 2 \mathrm{mg} / \mathrm{kg}$, i.p.) and received preemptive analgesia (ketoprofen, $5 \mathrm{mg} / \mathrm{kg}$, s.c.) and local anesthesia (5 mg/kg lidocaine, $2 \mathrm{mg} / \mathrm{kg}$ bupivacaine, s.c.). This pharmacological 
combination, preemptive analgesia, and addition of bupivacaine in local anesthesia for the management of postsurgical pain resulted in reduction of weight loss and normal feeding behavior after surgery.

Using a stereotaxic apparatus (ASI instruments), a CMA-12 guide cannula was placed into the ventral hippocampus (A: -5.20 $\mathrm{mm}$; L: $+4.80 \mathrm{~mm}$; V: $-4.50 \mathrm{~mm}$ relative to bregma; HIP groups) or medium prefrontal cortex (A: $+3.2 \mathrm{~mm}, \mathrm{~L}:+0.8 \mathrm{~mm}, \mathrm{~V}:-5.2 \mathrm{~mm}$ relative to bregma; mPFC groups) (Paxinos and Watson, 2014). A Silastic medical-grade tubing (Dow Corning) was inserted in the right jugular vein and passed subcutaneously to the posterior surface of the neck for total blood sampling. To avoid clotting, the jugular vein catheter was filled with heparin solution $(100 \mathrm{IU} / \mathrm{ml}$ in phosphate buffer saline, $\mathrm{pH} 7.4 \pm 0.1$ ). Animals recovered from the surgery in individual polypropylene boxes with food and water ad libitum.

In Vivo Microdialysis Probe Recovery. QTP in vivo relative recovery was determined by retrodialysis $\left(R_{R D}\right)$ in HIP and mPFC. After the surgery recovery period and 30 minutes before the start of the experiments, male and female ( $n=4 /$ brain region/sex) SCZ-like animals were placed in a CMA/120 system for freely moving animals, and the guide cannula was carefully replaced with a CMA/12 microdialysis probe ( $3 \mathrm{~mm}$, PAES membrane, $20 \mathrm{kDa}$ cutoff; CMA, Sweden). The probe was perfused with QTP $300 \mathrm{ng} / \mathrm{ml}$ solution in ACF at a flow rate of $2.0 \mu \mathrm{l} / \mathrm{min}$ for 1 hour for equilibration. Microdialysate samples were collected each 30 minutes up to 120 minutes, and $35 \mu l$ was injected directly into the liquid chromatography system for quantification. In vivo $R_{R D}$ was calculated as described previously (Araújo et al., 2008).

Probe relative recovery in vivo did not differ between male and female rats, and the mean value of $28.1 \% \pm 5.3 \%$ and $31.1 \% \pm$ $6.1 \%$ and for HIP and $\mathrm{mPFC}$, respectively, was used to determine real QTP free brain concentrations.

Quetiapine Plasma Pharmacokinetic and Unbound HIP and mPFC Distribution. SCZ-like offspring were randomly divided into the following eight groups according to sex and treatment type: $\mathrm{FQ}_{\text {HIP, male }}(n=8), \mathrm{QLNC}_{\mathrm{HIP}}$, male $(n=7), \mathrm{FQ}_{\text {HIP, female }}(n=7)$, $\mathrm{QLNC}_{\mathrm{HIP}}$, female $(n=6), \mathrm{FQ}_{\mathrm{mPFC}}$, male $(n=6), \mathrm{QLNC}_{\mathrm{mPFC}}$, male $(n=6)$, $\mathrm{FQ}_{\mathrm{mPFC}}$, female $(n=6)$, and $\mathrm{QLNC}_{\mathrm{mPFC}}$, female $(n=6)$. Naive offspring were also divided into the following groups: $\mathrm{FQ}_{\text {naive,male, } \operatorname{mPFC}}(n=6)$, $\mathrm{QLNC}_{\text {naive,male, } \mathrm{mPFC}}(n=6)$, and $\mathrm{FQ}_{\text {naive,female,HIP }}(n=6)$.

To allow model building, QTP total plasma and unbound HIP concentrations determined by microdialysis previously reported, obtained from experiments in awake male naive groups that received $\mathrm{FQ} \mathrm{(5} \mathrm{and} 10 \mathrm{mg} / \mathrm{kg}$ doses, i.v.) or QLNC ( $5 \mathrm{mg} / \mathrm{kg}$, i.v.), were employed ( $n=6$ to 7 animals/group) (Carreño et al., 2016a,b). Table 1 summarizes all the pharmacokinetic groups from the present work and from previously published data. Fig 1 summarizes the study desing used in the present work
QTP total plasma pharmacokinetics and unbound brain concentrations, determined by microdialysis, were evaluated in awake animals after a single intravenous bolus dose via lateral tail vein of QTP solution (FQ groups, $10 \mathrm{mg} / \mathrm{kg}$ ) or QLNC formulation (QLNC groups, $5 \mathrm{mg} / \mathrm{kg}$ ). It is worth noting that total plasma concentrations have different meanings after FQ and QLNC; for the latter, they represent the sum of the encapsulated drug plus the fractions unbound and bound to proteins in plasma.

On the day of the experiment, animals were placed individually in a CMA-120 system for freely moving animals, and the guide cannula was replaced with a previously calibrated CMA/12 probe for microdialysis sampling. The microdialysis system was stabilized for 1 hour with perfusion of ACF solution at a flow rate of $2.0 \mu \mathrm{l} / \mathrm{min}$. After animal dosing, microdialysate samples were collected every 30 minutes for up to 8 hours. A previously validated HPLC-UV method was used to analyze microdialysate samples $(35 \mu \mathrm{l})$ that were injected directly into the system without any processing (Carreño et al., 2016b). More information regarding model validation as well as precision and accuracy of the microdialysis method is described in Supplemental Material (Supplemental Fig. 1; Supplemental Tables 1 and 2).

Blood samples were collected through the jugular vein at scheduled time points $(0.083,0.25,0.5,1,2,4,6$, and 8 hours after dosing). Plasma obtained by centrifugation $\left(1300 \mathrm{~g}, 10\right.$ minutes, at $\left.4 \pm 2^{\circ} \mathrm{C}\right)$ was stored at $-80 \pm 1^{\circ} \mathrm{C}$ until analysis. A previously validated HPLC-UV method was used for the quantification of QTP in plasma samples (Carreño et al., 2016a).

\section{Data Analysis}

Data were analyzed based on the population analysis approach using the software NONMEM (version 7.4; ICON Development Solutions, Ellicott City, MD) with the first-order conditional estimation method and INTERACTION. Values of concentration observed were logarithmically transformed for the analysis. Data below the quantification limit were reported and were not included in the data set because they represented less than $5 \%$ of the total. Interindividual variability (IIV) was modeled exponentially, and the nondiagonal elements of the $\Omega$ variance-covariance matrix were evaluated for significance. Residual variability was described with an additive error model on the logarithmic scale.

Model building was performed sequentially. A model describing the disposition of unbound and total quetiapine concentrations in plasma and brain after single intravenous administration of FQ to naive and SCZ-like animals was first developed. Then, the model was expanded, integrating plasma and brain disposition data of quetiapine after injection of QLNC to naive and SCZ-like rats.

Model Selection. Selection between different model candidates was performed according to the following criteria: 1) changes in the minimum value of the objective function approximately equal to

TABLE 1

Pharmacokinetic groups used during model development

\begin{tabular}{|c|c|c|c|c|c|}
\hline Pharmacokinetic Group & Formulation, Dose & Number of Animals & Sex & Disease Status & Reference \\
\hline \multirow[t]{6}{*}{ Total plasma and unbound hippocampus } & $\mathrm{FQ}, 5 \mathrm{mg} / \mathrm{kg}$ & 5 & Male & Naïve & Carreño et al., $2016 \mathrm{~b}$ \\
\hline & $\mathrm{FQ}, 10 \mathrm{mg} / \mathrm{kg}$ & 6 & Male & Naïve & Carreño et al., 2016b \\
\hline & & 6 & Female & Naïve & Present work \\
\hline & $\mathrm{QLNC}, 5 \mathrm{mg} / \mathrm{kg}$ & 6 & Male & Naïve & Carreño et al., 2016b \\
\hline & & 7 & Male & SCZ-like & Present work \\
\hline & & 6 & Female & SCZ-like & Present work \\
\hline \multirow[t]{6}{*}{ Total plasma and unbound mPFC } & $\mathrm{FQ}, 10 \mathrm{mg} / \mathrm{kg}$ & 6 & Male & Naïve & Present work \\
\hline & & 6 & Male & SCZ-like & Present work \\
\hline & & 6 & Female & SCZ-like & Present work \\
\hline & $\mathrm{QLNC}, 5 \mathrm{mg} / \mathrm{kg}$ & 6 & Male & Naïve & Present work \\
\hline & & 6 & Male & SCZ-like & Present work \\
\hline & & 6 & Female & SCZ-like & Present work \\
\hline
\end{tabular}




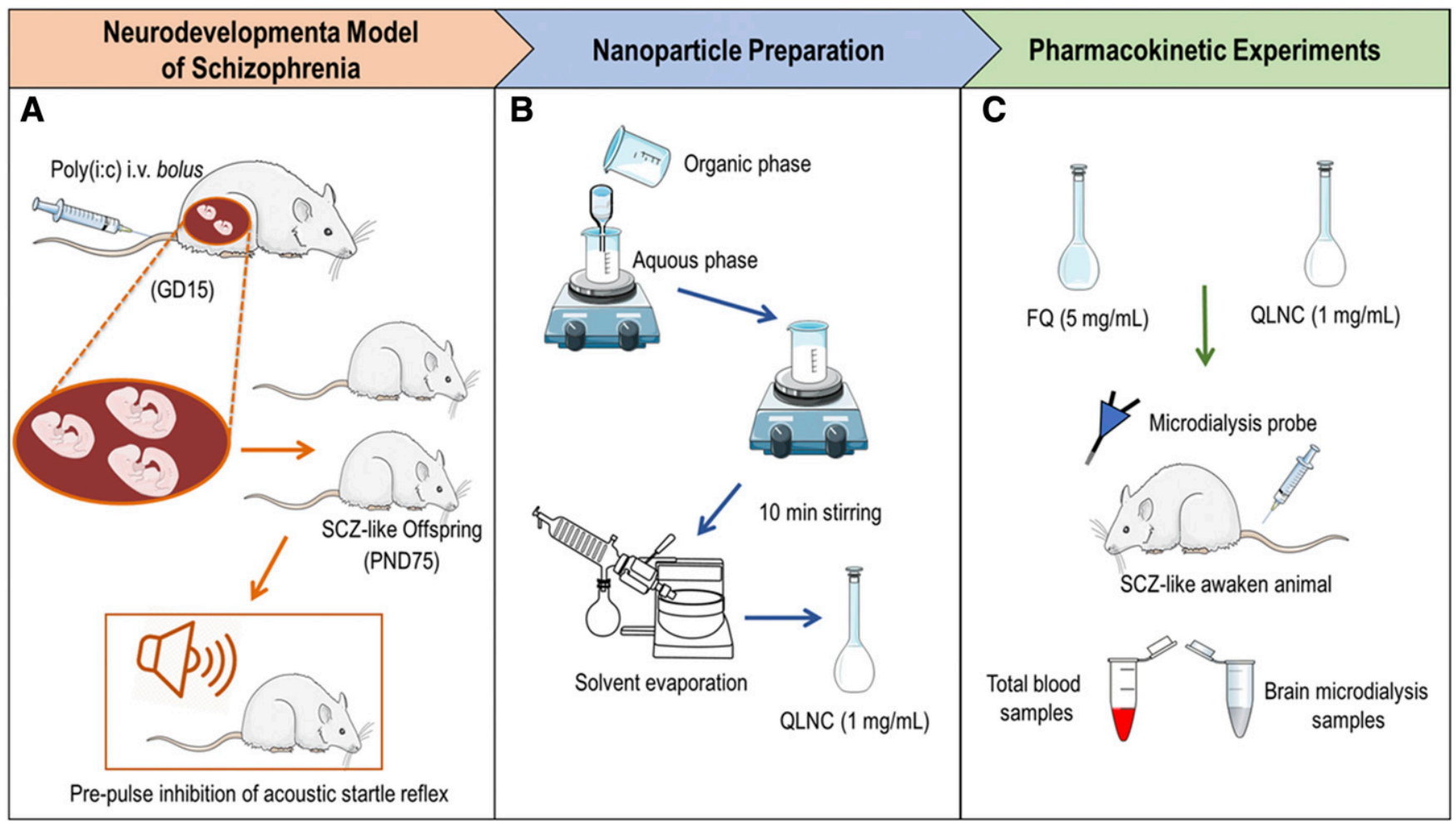

Fig. 1. Schematic representation of the pharmacokinetic study design performed in the present work. (A) SCZ-like offspring were obtained from pregnant dams (GD15) that received a $4 \mathrm{mg} / \mathrm{kg}$ i.v. bolus dose of poly(i:c). (B) QLNC formulation was prepared according to that previously published. (C) Total QTP plasma and unbound brain (hippocampal and $\mathrm{mPFC}$ ) concentrations were determined after intravenous dosing of quetiapine solution (FQ) or quetiapine loaded to lipid core nanocapsules (QLNC) to SCZ-like and naive rats.

$-2 \times \log$ (likelihood) (for nested models, the addition of a parameter was considered statistically significant when value of the objective was reduced by 3.84 or 6.64 points; $P<0.05$ or $P<0.01$, respectively for one degree of freedom), 2) visual exploration of goodness-of-fit plots, and 3) precision of model parameters reflected by the relative S.E. (RSE), computed as the ratio between the S.E. and the parameter estimate.

Model Evaluation. For each formulation and experimental group, 1000 simulated PK animal profiles were generated; then, for each measurement time, the 5th, 50th, and 95th percentiles were calculated and displayed graphically together with the experimental data. Parameter precision was further evaluated from the analysis of 500 bootstrap data sets.

Model-Based Exploration. Detailed comparison of the influence of the chronic schizophrenia disease on the systemic and brain exposures of quetiapine in both formulations was obtained via deterministic simulations from the selected model. In addition, the impact on the mentioned drug exposures of key parameters of the models affecting the pass through the blood brain barrier and drug release from the nanodevice will be explored using model-based simulations as well.

Pharmacokinetic Models.

FQ formulation. Pharmacokinetics of unbound concentrations of quetiapine in plasma $\left(\mathrm{C}_{\mathrm{u}, \mathrm{p}}\right)$ and in brain $\left(\mathrm{C}_{\mathrm{u}, \mathrm{B}}\right)$ were described using compartmental models parameterized in apparent volumes of distribution, distribution clearances, and total elimination clearance (CL). Models considering one, two, and three compartments were tested for both systemic and brain disposition of quetiapine. Total plasma drug concentrations $\left(\mathrm{C}_{\mathrm{P}}\right)$ were described as $\mathrm{C}_{\mathrm{u}, \mathrm{P}}$ divided by the quetiapine unbound fraction in plasma $\left(f_{u}\right)$.

Reversible transfer of unbound drug between central compartment and brain through the $\mathrm{BBB}$ were described by the distribution clearances $\mathrm{CL}_{\mathrm{in}}$ and $\mathrm{CL}_{\text {out }}$ equal to $\mathrm{k}_{\text {in }} \times \mathrm{V}_{1}$ and $\mathrm{k}_{\text {out }} \times \mathrm{V}_{\mathrm{u} \text {,Brain }}$, where $K_{\text {in }}$ and $K_{\text {out }}$ are the first-order rate constants of influx and efflux, respectively. $V_{1}$ is the apparent volume of distribution of the central compartment. The value of $\mathrm{V}_{\mathrm{u}, \text { Brain }}$, the brain volume of distribution for unbound QTP, used in the current analysis was $3.4 \times 10^{-4} \mathrm{l}$, calculated based on our previous work (Carreño et al., 2016a) according to the approach described by Tunblad et al. (2004).

QLNC formulation. It has been assumed that once quetiapine is released from the $\mathrm{LNC}$, the corresponding time courses of $\mathrm{C}_{\mathrm{u}, \mathrm{P}}, \mathrm{C}_{\mathrm{u}, \mathrm{B}}$, and $\mathrm{C}_{\mathrm{P}}$ are described by the model developed for the $\mathrm{FQ}$ as explained above. Therefore, data following LNC dosing provide support to characterize the released kinetics in different tissues and distribution characteristics of the nanocarrier.

During model building, the possibility that partition of quetiapine within LNC is not homogenous was also evaluated. In particular, the case in which fractions of the administered dose were assumed to be located in the core $\left(\mathrm{F}_{\text {core }}\right)$ or in the external layers $\left(\mathrm{F}_{\text {external }}\right)$ of the nanocarrier are released into the central unbound QTP compartment at first-order rates described by the constants $\mathrm{K}_{\mathrm{REL}}$, Core and $\mathrm{K}_{\mathrm{REL}}$, Ext, respectively, and remaining dose fraction $\left(\mathrm{F}_{\text {Surface }}\right)$, assumed to be nonencapsulated, enters the systemic circulation directly as FQ.

Dispositions of QLNC were also characterized with compartmental models as described above for the case of the FQ solution, assuming that quetiapine could only be eliminated once it has been released from the nanoparticle.

Unbound concentrations in the microdialysate samples were described by the integral over each collection interval instead of a midpoint approach (Tunblad et al., 2004), as will be mathematically described in the next section. All models consisted of algebraically and ordinary differential equations.

The presence of nonlinear concentration-dependent kinetics was also investigated during the course of the analysis, and finally the impact of the poly(i:c) exposure, weight, and gender in all model parameters was investigated with stepwise covariate modeling, using a forward inclusion $(P<0.05)$ followed by backward elimination process $(P<0.01)$ (Wählby et al., 2002). 
Softwares. The PsN (version 4.5.1) tool kit (Lindbom et al., 2005), the $\mathrm{R}$ (version 3.5.1) program, and the Xpose 4 (version 4.4.1) program (Jonsson and Karlsson, 1999) were used for automating and controlling the runs, data visualization, and graphical analysis (goodness-of-fit graphics, including predicted-corrected visual predictive check and bootstrap analysis), respectively, as well as stepwise covariate modeling. The Pirana (version 2.9.8) program (Keizer et al., 2013) was used to keep track of run records and results.

\section{Results}

Prepulse Inhibition of Acoustic Startle Reflex. The mean PPI (\%) across all prepulse stimuli used for naive and SCZ-like animals agree with our previous study (Carreño et al., 2020). Adult SCZ-like offspring exhibited significant preattentional sensorimotor gating deficits $(P<0.01)$ manifested as a reduction in PPI (Supplemental Fig. 1).

Population Pharmacokinetic Analysis. A total of 1158 observations (356 total plasma, 42 unbound plasma, and 760 brain microdialysate samples) from a total of 56 rats were used for the analysis of the $\mathrm{FQ}$ formulation. With respect to the QLNC formulation, data were obtained from 42 rats and comprised 886 observations (296 total plasma, 72 unbound plasma, and 518 brain microdialysate samples), including data from previous studies.

FQ Formulation. A two-compartment model better described $\mathrm{C}_{\mathrm{u}, \mathrm{P}}$ pharmacokinetics than a one-compartment model $(P<0.01)$. Increasing the number of distribution compartments did not improved the fit significantly $(P>0.05)$. The apparent volumes of distributions of the two compartments were represented by $\mathrm{V}_{1, \mathrm{u}}$ and $\mathrm{V}_{2, \mathrm{u}}$ (peripheral) and the distribution and elimination clearances by $\mathrm{Q}_{\mathrm{u}}$ and $\mathrm{CL}_{\mathrm{u}}$, respectively

Reversible transfer of unbound quetiapine between central compartment and brain through the $\mathrm{BBB}$ were characterized by the distribution clearances $\mathrm{CL}_{\mathrm{D} \text {,in }}$ and $\mathrm{CL}_{\mathrm{D} \text {,out }}$ as stated before in the Material and Methods section. Attempts to describe that distribution process with equal values of $C L_{D \text {,in }}$ and $C L_{D, \text { out }}$ resulted in a significantly worse fit $(P<0.01)$.

Disposition of unbound quetiapine concentrations in brain $\left(\mathrm{C}_{\mathrm{u}, \mathrm{B}}\right)$ was significantly better described with a twocompartment model in comparison with a model with just one compartment $(P<0.01)$. The cerebrospinal space was considered as the peripheral compartment within brain, with values of volume of distribution $\left(\mathrm{V}_{\mathrm{CSF}}\right)$ of $2.5 \times 10^{-4} \mathrm{l}$ (calculated as described for $\mathrm{V}_{\mathrm{u} \text {,Brain }}$ ) and bulk flow ( $\mathrm{Q}_{\mathrm{Bulk}}$ ) of $1.2 \times 10^{-5} \mathrm{l} / \mathrm{h}^{21}$. Figure $2 \mathrm{~A}$ provides the schematic representation of the PK model selected for quetiapine injected through the FQ.

Pharmacokinetic Model after the Administration of the LNC. The model, considering that quetiapine in the LNC is located in the core, in the external layers, and part adsorbed to the surface, where $\mathrm{F}_{\text {core }}, \mathrm{F}_{\text {nonencapsulated }}$, and $\mathrm{F}_{\text {surface }}$ represent the corresponding fractions of the administered dose, performed significantly better $(P<0.001)$ than other options, such as those differing just between core and surface or assuming homogenous distribution.

Drug release from the core and external layers were best described through first-order processes governed by the firstorder rate constants $\mathrm{k}_{\text {core }}$ and $\mathrm{k}_{\text {external, }}$, respectively. The amount of drug adsorbed to the surface entered the systemic circulation directly as it was the case of $\mathrm{FQ}$ formulation.
Models describing the release process with zero-order kinetics performed significantly worse $(P<0.001)$.

Data did not support distribution of the quetiapine embedded in the external layers to brain and peripheral compartments and irreversible elimination $(P>0.05)$, and therefore its pharmacokinetics were fully characterized by $\mathrm{F}_{\text {external, }}$, $\mathrm{k}_{\text {external }}$, and $\mathrm{V}_{1 \text {,nano, }}$, the apparent volume of distribution of the quetiapine encapsulated in the central compartment.

With respect to the drug in the core of the nanocarrier, the model could identify distribution from the central compartment to the peripheral compartment and brain but not vice versa; in fact, when bidirectional transfer between compartments was tested, a worse description of the data were obtained. $Q_{\text {nano }}$ represents the distribution clearance between the central compartment and both the brain and peripheral compartments, as the distinction between tissues in the parameter were nonsignificant $(P>0.05)$.

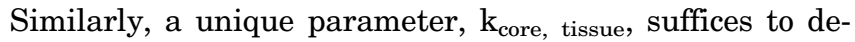
scribe the first-order rate of release of quetiapine from the core of the nanocarrier into either the brain or peripheral compartments. The apparent volume of distribution of the central compartment of the nanocarriers incorporating quetiapine in their core was also $V_{1, \text { nano. }}$. Data did not support an elimination process of quetiapine encapsulated $(P>0.05)$. In addition, the two-compartment brain model found for $\mathrm{C}_{\mathrm{u}, \mathrm{B}}$ was not supported for quetiapine in the core of the nanodevice $(P>0.05)$.

The schematic representation of the integrated model capable to describe QTP plasma and brain concentrationtime profiles given either as FQ or LNC is shown in Fig. 2B. The model represented mathematically by the set of ordinary differentials below expands the model described in Supplemental Material:

$$
\begin{aligned}
& \frac{d A_{\text {nano,core }}}{d t}=-\left(\frac{Q_{\text {nano }}}{V_{1, \text { NANO }}}+\frac{Q_{\text {nano }}}{V_{1, \text { NANO }}}+K_{\text {core }}\right) \cdot A_{1} \\
& \frac{d A_{u, p}}{d t}=-\left(\frac{C L_{u}}{V_{1}}+\frac{Q_{u}}{V_{1}}+\frac{C L_{\text {in }}}{V_{1}}\right) \cdot A_{2}+\frac{C L_{\text {out }}}{V_{u, \text { brain }}} \cdot A_{6}+K_{\text {core }} \cdot A_{1} \\
& +K_{\text {external }} \cdot A_{3} \\
& \frac{d A_{\text {nano,external }}}{d t}=-K_{\text {external }} \cdot A_{3} \\
& \frac{d A_{\text {nano } p}}{d t}=-K_{\text {core }, \text { tissue }} \cdot A_{4}+\frac{Q_{\text {nano }}}{V_{1, \text { NANO }}} \cdot A_{1} \\
& \frac{d A_{\text {nano,tissue }}}{d t}=-\frac{Q_{u}}{V_{2}} \cdot A_{5}+K_{\text {core,tissue }} \cdot A_{4}+\frac{Q_{u}}{V_{1}} \cdot A_{2} \\
& \frac{d A_{u, B}}{d t}=-\left(\frac{C L_{D, \text { out }}}{V_{u, \text { Brain }}}+\frac{Q_{\text {bulk }}}{V_{u, \text { Brain }}}\right) \cdot A_{6}+\frac{C L_{D, \text { in }}}{V_{1}} \cdot A_{2}+K_{\text {core,tissue }} \cdot A_{7} \\
& +\frac{Q_{b u l k}}{V_{C S F}} \cdot A_{8} \\
& \frac{d A_{\text {nano }, B}}{d t}=-K_{\text {core }, \text { tissue }} \cdot A_{7}+\frac{Q_{\text {nano }}}{V_{1, \text { NANO }}} \cdot A_{1} \\
& \frac{d A_{C S F}}{d t}=-\frac{Q_{b u l k}}{V_{C S F}} \cdot A_{8}+\frac{Q_{b u l k}}{V_{u, b r a i n}} \cdot A_{6} .
\end{aligned}
$$

$\mathrm{A}_{\mathrm{u}, \mathrm{p}}, \mathrm{A}_{\mathrm{u}, \mathrm{T}}, \mathrm{A}_{\mathrm{u}, \mathrm{B}}$, and $\mathrm{A}_{\mathrm{CSF}}$ are unbound amounts of quetiapine in the central, peripheral, brain, and cerebrospinal compartments, respectively; the amount of QTP loaded into the nanocarrier in its central, peripheral, and brain 
compartments are represented by $A_{\text {nano,p }}, A_{\text {nano, tissue, and }}$ $\mathrm{A}_{\text {nano,B, }}$, respectively.

The quetiapine encapsulated concentrations were calculated as $\left(A_{\text {nano, core }}+A_{\text {nano,p }}\right) / V_{1, \text { NANO }}$.

Covariate Analysis. Parameters $\mathrm{CL}_{\mathrm{D} \text {,in }}$ and $\mathrm{CL}_{\mathrm{D} \text {,out }}$ were significantly impacted by both Schizophrenia status and \%PPI $(P<0.001)$ as represented by eq. 11 below; however, neither weight nor gender significantly influenced any of the model's parameters $(P>0.05)$ :

$$
\begin{aligned}
C L_{D, \text { in } / \text { out }}= & {\left[\theta_{C L D_{\text {in }} / \text { out } \_S C Z} \times S C Z+\theta_{C L D_{\text {in } / \text { out } \_ \text {naö̀ve }}} \times(1-S C Z)\right] } \\
& \times \frac{\% P P I}{\% P P I_{m d}}
\end{aligned}
$$

where SCZ takes values of 1 and 0 for SCZ-like and naive animals, respectively; $\% \mathrm{PPI}_{\mathrm{md}}$ is the median of the \%PPI values, and $\theta_{\text {CLDin/out_i }}$ and $\theta_{\text {CLDin/out_i }}$ represent the values for $\mathrm{CL}_{\mathrm{D} \text {,in }}$ and $\mathrm{CL}_{\mathrm{D} \text {,out }}$ corresponding (i, either SCZ-like or naive) to a value of \%PPI equal to its median. Supplemental Figure 1

A

BBB

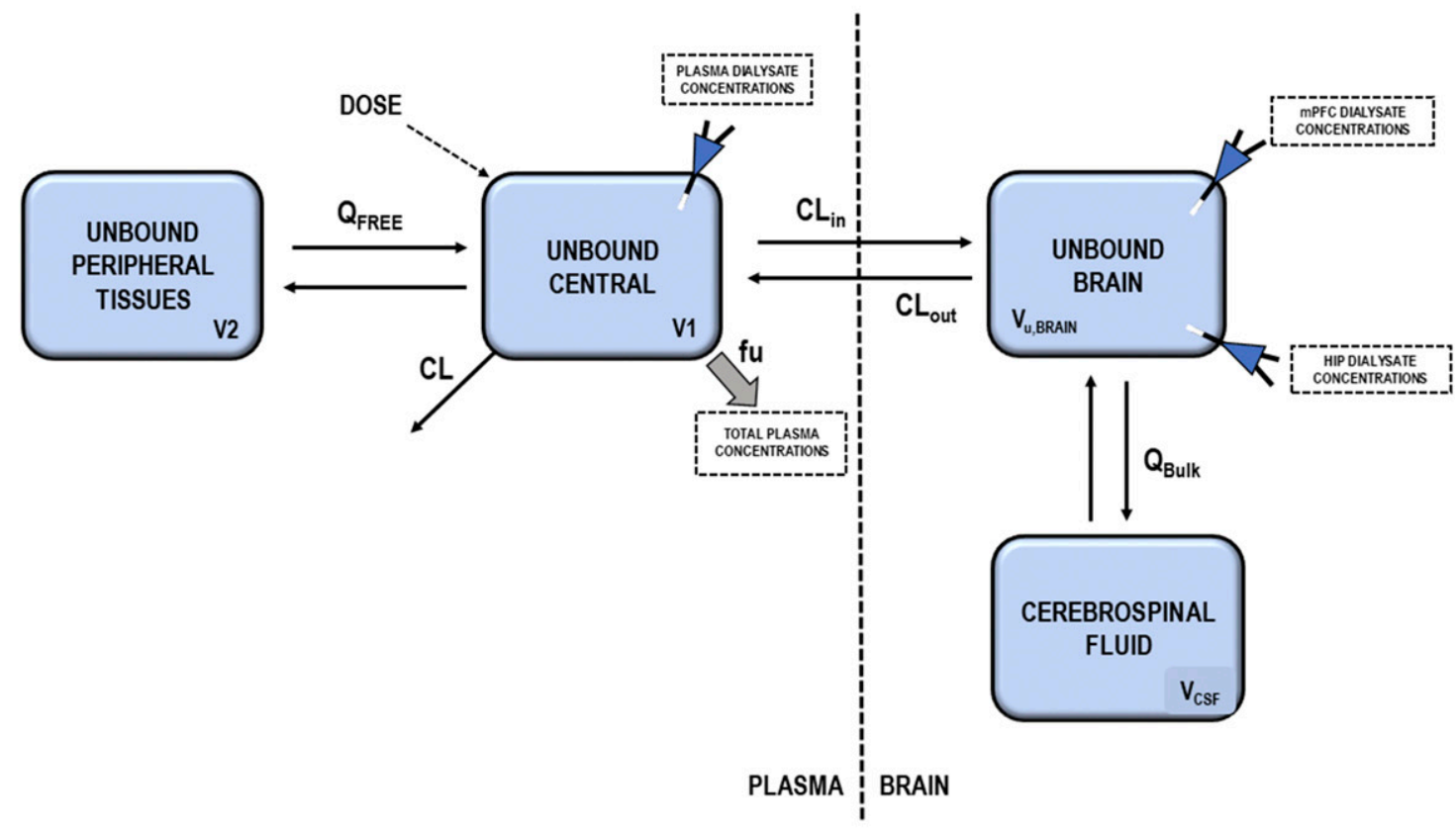

B

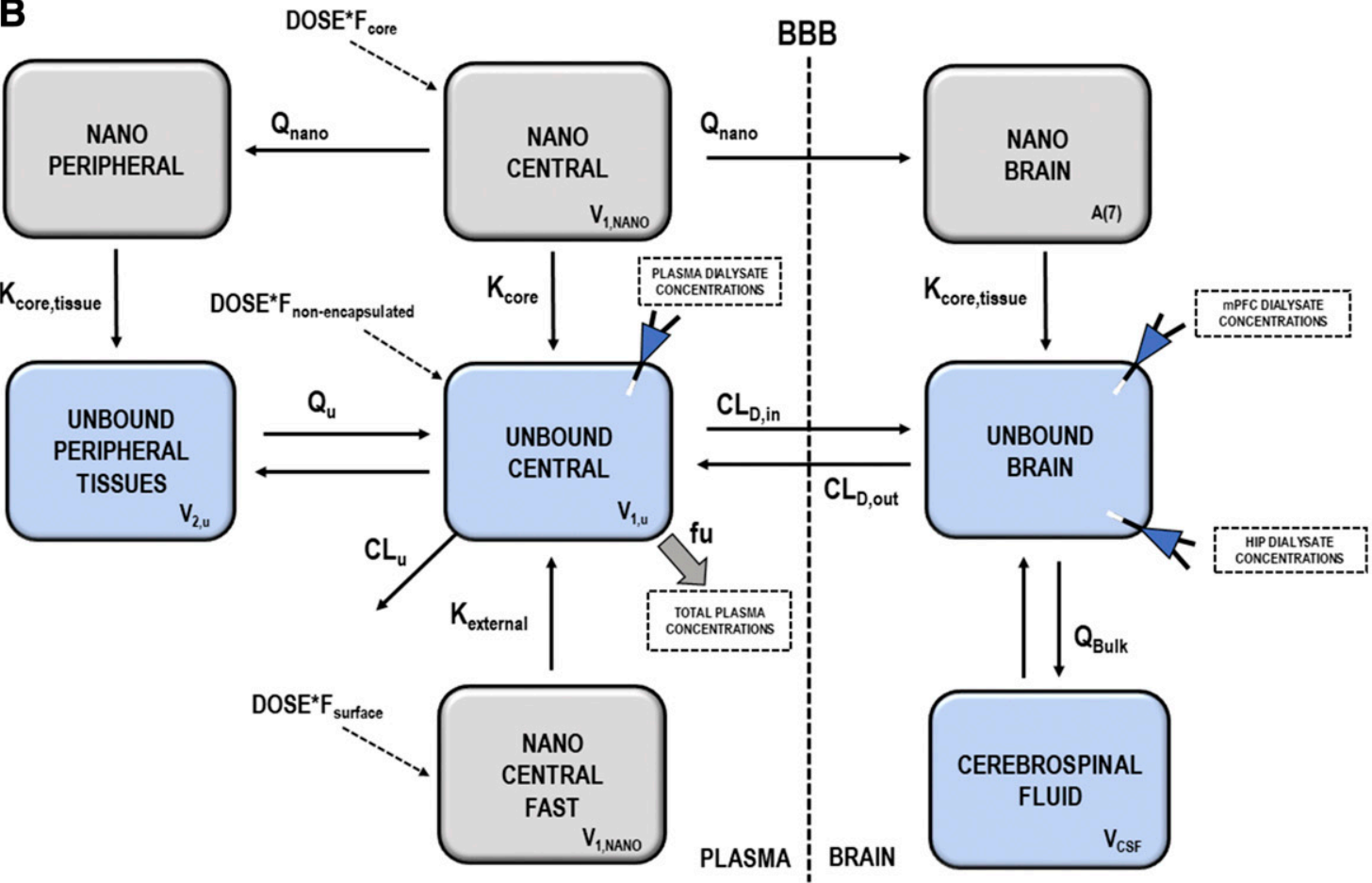

Fig. 2. Schematic representation of the final model developed for quetiapine administered intravenously in FQ (A) and LNC (B) formulations. All parameters and terms are defined in the text. 
shows the relationship between either $\mathrm{CL}_{\mathrm{D} \text {,in }}$ or $\mathrm{CL}_{\mathrm{D} \text {,out }}$ versus \%PPI.

Model Parameter Estimates and Model Evaluation. Table 2 lists the estimates of the parameters of the final model. Parameters were precisely estimated, and neither were any of the \%RSEs greater than $35 \%$ nor the lower limit of the 95th confidence intervals calculated from the bootstrap analysis lower or equal zero. Figure 3 shows the results from the simulation-based diagnostics, in which for all experimental conditions (unbound and total plasma concentrations, both formulations, and naive and SCZ-like animals), the median tendency as well as the dispersion of the data were very well captured by the model. Individual observations and model predictions versus time profiles are shown in Supplemental Figs. 4-6. Goodness-of-fit plots are presented in Supplementary Figs. 7 and 8 and did not reveal model misspecifications.

The fractions of the dose of quetiapine located in the core, adsorbed in the surface, and in the external layer of the nanocarriers were $0.85,7$, and 8 , the latter derived as $\left[1-\left(\mathrm{F}_{\text {core }}+\mathrm{F}_{\text {surface }}\right)\right]$. Remarkably, the first-order constant accounting for the rate of drug release from the core $\left(\mathrm{k}_{\text {core }}\right)$ into the systemic circulation was estimated much lower $\left(4.7 \times 10^{-4}\right.$ hour $^{-1}$ ) with respect to 1 ) the same process occurring in the peripheral compartment and in the brain $\left(\mathrm{k}_{\text {core, tissue }}=\right.$ 0.261 hour $^{-1}$ ) and 2) the release from the external layer $\left(\mathrm{k}_{\text {external }}=0.0836\right.$ hour $\left.^{-1}\right)$.

Distribution clearances of unbound quetiapine and LNC corresponding to brain distribution were of the same order of magnitude and greater for LNC. Chronic schizophrenia reduced a $\mathrm{CL}_{\mathrm{D} \text {,in }}$ and $\mathrm{CL}_{\mathrm{D} \text {,out }}$ by $58 \%$ and $24 \%$, respectively. The estimates of $\mathrm{CL}_{\mathrm{D} \text {,in }}$ and $\mathrm{CL}_{\mathrm{D} \text {,out }}$ were quite similar in SCZ-like animals, whereas in naive rats, $\mathrm{CL}_{\mathrm{D} \text {,in }}$ was twice the estimate of $\mathrm{CL}_{\mathrm{D} \text {,out. }}$. Apparent volumes of distribution of unbound quetiapine are higher than the volume of physiologic water. On the other hand, the apparent volume of distribution of the central compartment of quetiapine in the nanocarrier was slighted lower than the volume of physiologic water. Finally, the estimate of $f_{u}$ was 0.24 .
Data supported the estimation of IIV in CL and in the parameters governing the bidirectional transport across BBB of unbound QTP. The magnitudes of IIV ranged from $17 \%$ to $67 \%$. The estimate in vivo $\mathrm{f}_{\mathrm{u}}$ was 0.24 .

Model Exploration and External Validation. Figure 4 shows the typical simulated concentration versus time profiles in plasma and in brain for both formulations studied in both type of animals, naive and SCZ-like. Simulation results indicate that LNC formulation increased quetiapine exposure in plasma in both groups of animals in comparison with the FQ solution. On the other hand, unbound brain concentrations after administration of FQ were half in SCZ-like animals compared with the naive group. Those differences were overcome when the LNC was given, as the typical simulated profiles show similar unbound concentrations in both groups.

The model developed for the FQ formulation was externally validated with a set of total plasma and unbound brain concentrations of quetiapine obtained from naive ( $n=6$ animals) animals receiving a single FQ $5 \mathrm{mg} / \mathrm{kg}$ i.v. dose and 30 minutes after, a $30 \mathrm{mg} / \mathrm{kg}$ i.v. dose of probenecid, an influx inhibitor (Carreño et al., 2016b). Typical parameter estimates listed in Supplemental Table 1 were remarkably similar to those listed in Table 1 , with the exception of $\mathrm{CL}_{\mathrm{D} \text {,in }}$, which was reduced from 0.045 (Table 2 ) to $0.028 \mathrm{l} / \mathrm{h}$ per kilogram; this is an expected result given the known effect of probenecid as an interacting drug and supporting the mechanistic interpretation of the parameters used to describe distribution of quetiapine in brain. Supplemental Figures 9 and 10 provide the individual observations and model predictions for total plasma and unbound brain concentrations of quetiapine as well as goodness-of-fit plots, respectively.

\section{Discussion}

Pathologic changes in $\mathrm{BBB}$ are recognized as a central factor for the development of several neurologic disorders (Lindqvist et al., 2013). Moreover, the high variability in chronic SCZ

TABLE 2

Pharmacokinetic parameters estimate of the selected integrated model for quetiapine

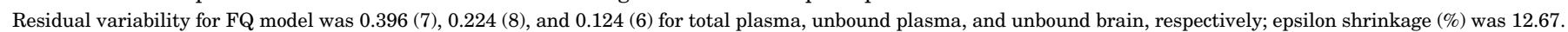

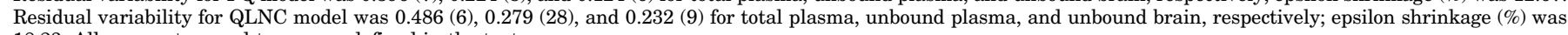
18.23. All parameters and terms are defined in the text.

\begin{tabular}{|c|c|c|c|c|c|}
\hline Parameters & $\begin{array}{l}\text { Estimate } \\
\text { (\% RSE) }\end{array}$ & $\begin{array}{l}\text { Median Bootstrap (5th-95th } \\
\text { Percentile) }\end{array}$ & IIV (\% RSE) & $\begin{array}{c}\text { Median Bootstrap IIV (5th-95th } \\
\text { Percentile) }\end{array}$ & Shrinkage (\%) \\
\hline $\mathrm{CL}_{\mathrm{u}}(\mathrm{l} / \mathrm{h}$ per kilogram $)$ & $1.86(16)$ & $1.79(1.56-1.92)$ & $22.9(2)$ & $(24.18-32.3)$ & 6.10 \\
\hline $\mathrm{V}_{1, \mathrm{u}}(\mathrm{l} / \mathrm{kg})$ & $1.31(8)$ & $1.32(1.21-1.34)$ & - & - & - \\
\hline $\mathrm{V}_{2, \mathrm{u}}(\mathrm{l} / \mathrm{kg})$ & $1.73(20)$ & $1.76(1.17-1.85)$ & - & - & - \\
\hline $\mathrm{Q}_{\mathrm{u}}(\mathrm{l} / \mathrm{h}$ per kilogram $)$ & $1.08(34)$ & $1.06(0.86-1.18)$ & - & - & - \\
\hline fu $(\%)$ & $24.2(12)$ & $23.9(21.78-25.8)$ & - & - & - \\
\hline $\mathrm{CL}_{\mathrm{D} \text {,in_naïve }}(\mathrm{l} / \mathrm{h}$ per kilogram $)$ & $0.045(10)$ & $0.043(0.031-0.049)$ & $16.7(12)$ & $19.35(15.45-22.3)$ & 17.3 \\
\hline $\begin{array}{l}\text { CL } \\
\text { kilout_naïve } \\
\text { kilogram })\end{array}$ & $0.023(14)$ & $0.021(0.018-0.027)$ & $26.4(23)$ & $24.3(20.35-30.3)$ & 21.3 \\
\hline $\mathrm{CL}_{\mathrm{D}, \text { in_SCZ }}(\mathrm{l} / \mathrm{h}$ per kilogram $)$ & $0.019(16)$ & $0.016(0.011-0.022)$ & $55.6(17)$ & $50.3(45.29-65.4)$ & 15.8 \\
\hline $\mathrm{CL}_{\mathrm{D}, \mathrm{out} \_\mathrm{SCZ}}(\mathrm{l} / \mathrm{h}$ per kilogram$)$ & $0.017(7)$ & $0.014(0.09-0.018)$ & $66.7(21)$ & $(60.32-76.1)$ & 32.4 \\
\hline $\mathrm{V}_{1, \text { nano }}(\mathrm{l} / \mathrm{kg})$ & $0.77(13)$ & $0.74(0.63-0.84)$ & - & - & - \\
\hline $\mathrm{k}_{\text {external }}\left(\mathrm{h}^{-1}\right)$ & $0.261(14)$ & $0.231(0.171-0.241)$ & - & - & - \\
\hline $\mathrm{k}_{\text {core }}\left(\times 10^{-4} \mathrm{~h}^{-1}\right)$ & $4.7(25)$ & $4.2(2.5-5.7)$ & - & - & - \\
\hline $\mathrm{k}_{\text {core, tissue }}\left(\mathrm{h}^{-1}\right)$ & $0.0836(35)$ & $0.0791(0.0461-0.0861)$ & - & - & - \\
\hline $\mathrm{Q}_{\text {nano }}(\mathrm{l} / \mathrm{h}$ per kilogram $)$ & $0.067(11)$ & $0.061(0.043-0.071)$ & - & - & - \\
\hline $\mathrm{F}_{\text {core }}(\%)$ & $85(11)$ & $84(75-86)$ & - & - & - \\
\hline $\mathrm{F}_{\text {surface }}(\%)$ & $7(36)$ & $5(3-8)$ & - & - & - \\
\hline $\mathrm{F}_{\text {external }}(\%)$ & $8^{a}$ & $7(5-9)$ & - & - & - \\
\hline
\end{tabular}

IIV, interindividual variability expressed as $\mathrm{CV}(\%)$ calculated as $\omega \times 100$, where $\omega$, is the S.D. of the variance of the random effect.

${ }^{a}$ Parameter derived as $\left[1-\left(\mathrm{F}_{\text {core }}+\mathrm{F}_{\text {surface }}\right)\right]$. 
A

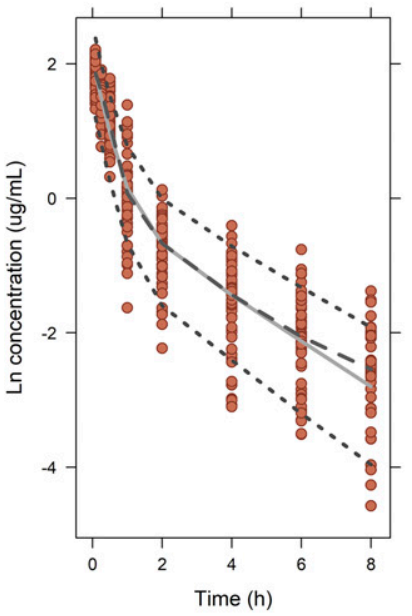

Naïve unbound brain

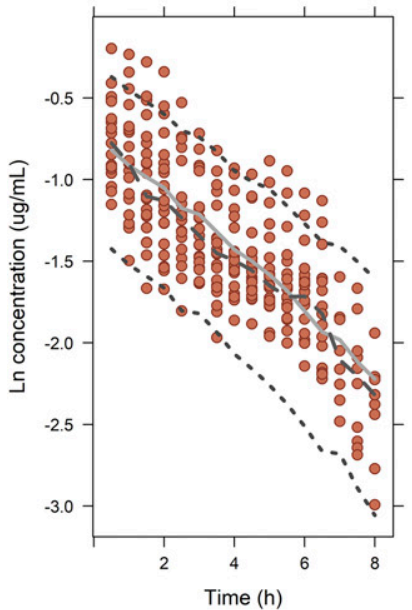

Unbound plasma

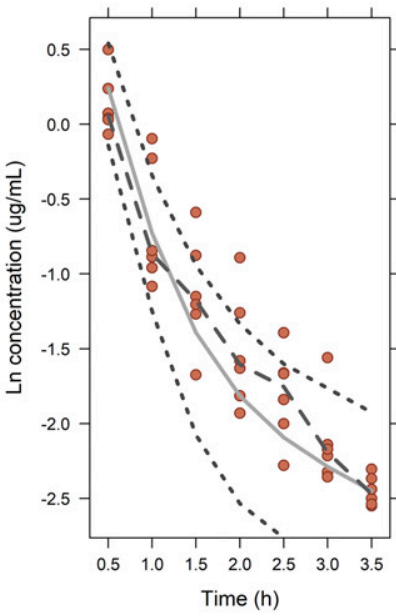

SCZ-like unbound brain

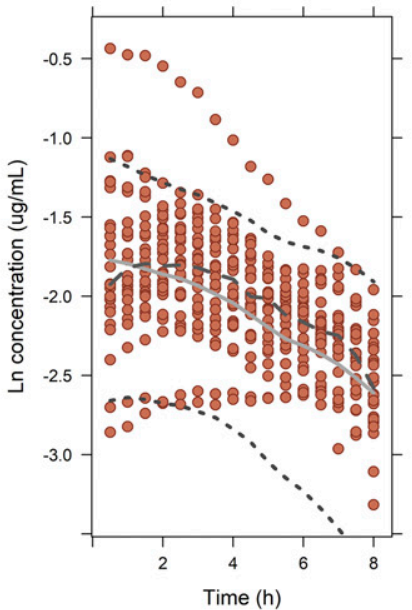

B
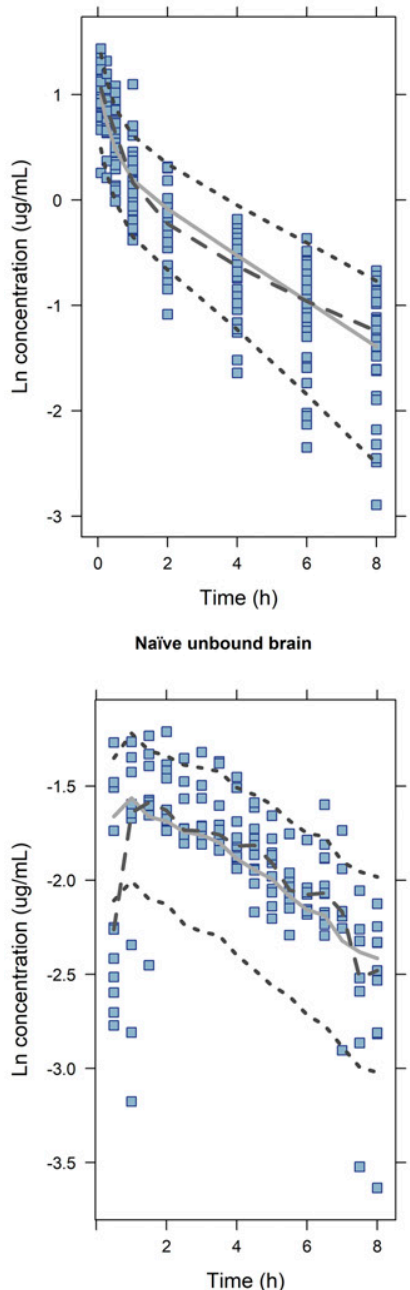

Unbound plasma

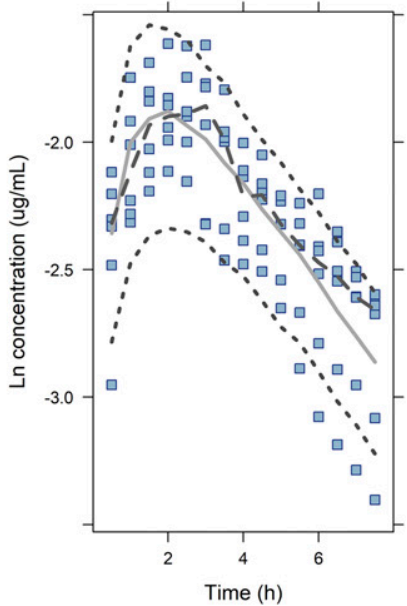

SCZ-like unbound brain

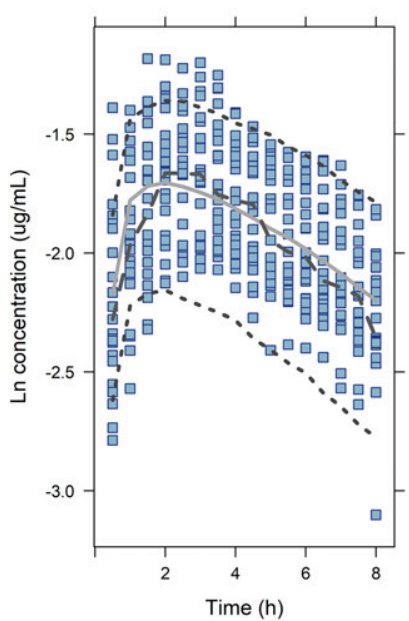

Fig. 3. Visual predictive checks of the final population PK model for (A) FQ and (B) QLNC formulations stratified by different experimental conditions. Panels show the result of 1000 simulations. Observations (dots and squares), median of the observations (thick solid lines), and dashed lines correspond to the 10th, 50th, and 90th percentiles of the simulated profiles.

response to treatment has been associated in part with $\mathrm{BBB}$ dysfunction. Limited drug delivery to CNS in schizophrenic patients has been suggested as a potential factor responsible of resistance to treatment observed in $30 \%$ of patients (Kennedy et al., 2014). Another intriguing finding reported in literature is the gender effects on brain drug penetration found in patients with chronic SCZ (Han et al., 2012). This work aimed to characterize, using a population pharmacokinetic modeling approach, QTP distribution through BBB of SCZ-like animals following administration of the drug nanoencapsulated in LNC, helping to elucidate the role of the delivery system in this disease scenario. From the pharmacokinetics perspective, the experimental framework was set up to allow the detection of possible factors causing treatment failure in individuals with SCZ.

No differences were found in any pharmacokinetic parameters for both formulations when data from male and female naive or SCZ-like animals were compared within the same formulation group. This result indicates that sex does not influence QTP plasma pharmacokinetics and brain penetration, even in the altered BBB condition investigated.

A significant reduction of the extent of QTP unbound brain distribution was observed in SCZ-like animals dosed with FQ when compared with naive offspring. Assuming $\mathrm{CL}_{\mathrm{D} \text {,in }} \mathrm{CL}_{\mathrm{D} \text {,out }}$ ratio as a measure of tissue penetration, the naive animals' ratio of 1.96, indicative of QTP influx to the brain, is reduced to 1.11 in SCZ-like animals, a decrease of about $43 \%$. The prenatal insult triggered by poly(i:c) impaired brain development of offspring, leading to a long-term alteration in BBB permeability of SCZ-like adult animals, mimicking what happens in humans, according to the neurodevelopmental hypothesis of SCZ.

The semimechanistic PK final model allowed for an understanding of the mechanisms underlying this reduced brain penetration, showing that SCZ-like animals present alterations in the influx transport across $\mathrm{BBB}$ represented by a reduction in the $\mathrm{CL}_{\mathrm{D} \text {,in }}$ (from 0.045 to $0.019 \mathrm{l} / \mathrm{h}$ per kilogram) when compared with the naive animals. Furthermore, using the PPI test, it was possible to demonstrate, for the first time, that the greater the preattentional sensorimotor gating deficit manifested as a reduction in PPI, the more compromised is the influx transport across BBB. Therefore, this result indicates that the cerebral penetration of antipsychotics such as QTP, which is an influx transport substrate, is decreased in SCZ and may contribute to the resistance observed with pharmacological treatment. 

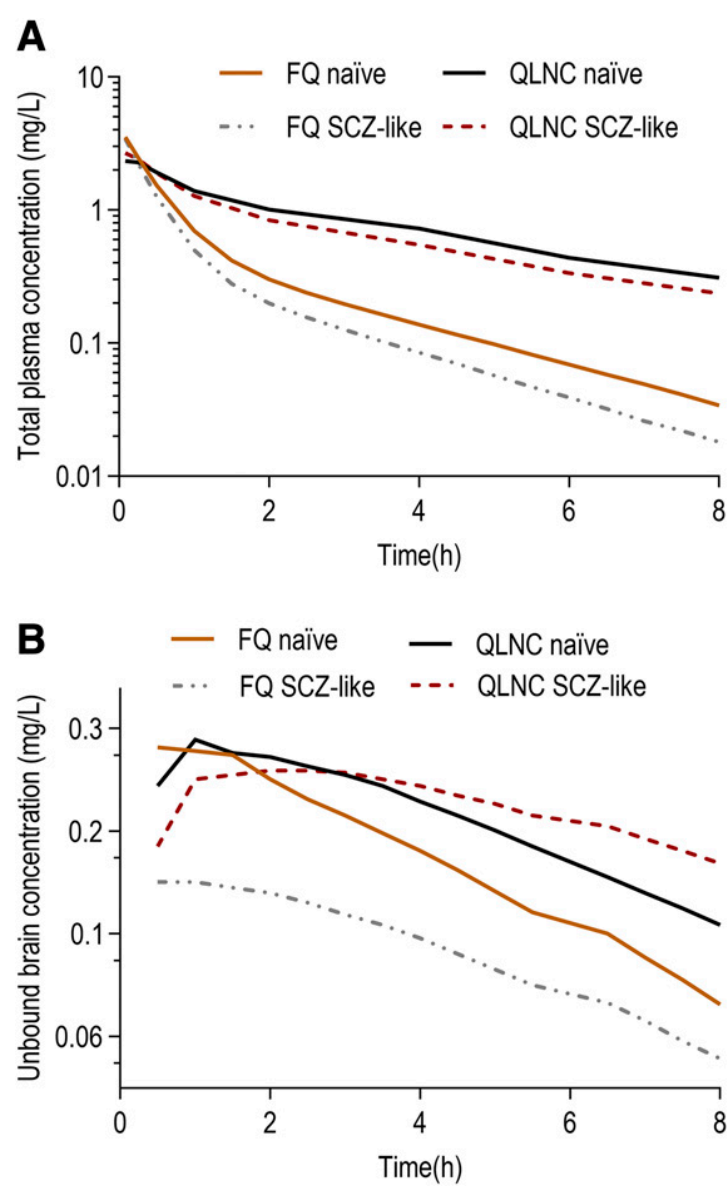

Fig. 4. Simulated time course of FQ and QLNC in plasma and brain of naive and SCZ-like rats. (A) QTP total plasma concentration-time profiles obtained after simulated intravenous bolus dosing of $5 \mathrm{mg} / \mathrm{kg}$ FQ or LNC. (B) QTP unbound brain concentration-time profiles obtained after simulated intravenous bolus dosing of $5 \mathrm{mg} / \mathrm{kg} \mathrm{FQ}$ or LNC.

To corroborate our hypothesis, the model was also applied to a different set of data obtained from naive animals (Carreño et al., 2016b) that receive QTP in association with the influx transporter inhibitor probenecid. During the analysis of these additional data, a reduction in $\mathrm{CL}_{\mathrm{D} \text {,in }}$ (Supplemental Table 1) of a magnitude very similar to that observed when comparing naive and SCZ-like animals (Table 1) was obtained, indicating that any alteration on influx transporter at BBB level will have a significant impact on QTP brain exposure.

Another hypothesis to explain this reduction in brain penetration of QTP in SCZ-like animals might be related to alteration of the efflux transport P-glycoprotein (P-gp) in the $\mathrm{BBB}$. The affinity of some atypical antipsychotic drugs such as quetiapine, risperidone, olanzapine, and clozapine in vitro by P-gp was examined by their P-gp ATPase activity, a putative measure of P-gp activity. Quetiapine and risperidone were found to be relatively good P-gp substrates, whereas olanzapine showed intermediate affinity and clozapine showed the least affinity of the drugs studied (Boulton et al., 2002).

According to the neurodevelopmental hypothesis of SCZ, exposure to inflammatory mediators in early life plays a role in SCZ and might influence P-gp expression or function, negatively influencing brain's permeability(Müller et al., 2015).

In humans, the P-gp activity was studied in vivo by positronemission tomography with [11C] verapamil, a P-gp substrate, in patients with chronic SCZ patients (de Klerk et al., 2010). The authors found a significant decrease of [11C] verapamil that correlates with an increased activity of the P-gp pump. Whether that is the case for the SCZ-like animals used in the present work remains to be investigated.

Our experimental setting demonstrated that QTP loaded into LNC modified drug delivery to the brain in naive and SCZ-like animals. Normally, the study of drug release kinetics from nanoparticles is carried out using in vitro data aiming to find a general model capable of describing a multimechanistic release. In the present study, using the model-based approach, we were able to describe the in vivo release kinetics of QTP from the QLNC in plasma and support a mechanism of nanocarrier distribution and drug release into the brain and other tissues.

QLNC shows drug-sustained release from the core to plasma, which is represented by the $\mathrm{K}_{\text {core }}$, and it is capable of maintaining the drug longer in the systemic circulation. However, the distribution clearance of the nanoparticle (Qnano) is faster than the release in plasma and governs the process of QTP distribution to tissues. A previous study using intravital microscopy demonstrated that LNCs act as drug shuttles through the BBB, delivering drugs on brain tissue with high efficiency after intravenous administration (Rodrigues et al., 2016).

The model-based approach described here provides an understanding on why, in naive animals, the rate of QTP distribution through the $\mathrm{BBB}$ when the drug is given as $\mathrm{FQ}$ is similar to that obtained when the drug is given as QLNC $\left(\mathrm{CL}_{\mathrm{D} \text {,in, }}\right.$, naive: $0.045 \mathrm{l} / \mathrm{h}$ per kilogram and $\mathrm{Q}_{\text {nano }} 0.067 \mathrm{l} / \mathrm{h}$ per kilogram). When the BBB is unaltered, the rate of nanocarrier brain penetration $\left(\mathrm{Q}_{\text {nano }}\right)$ governs $\mathrm{QTP}$ distribution and is like that observed for the unbound QTP brain penetration $\left(\mathrm{CL}_{\mathrm{D} \text {,in }}\right)$.

When SCZ-like animals are administered QLNC, part of the drug will be released in the blood $(\sim 15 \%)$, but the bigger amount will be carried across the BBB by the nanocarriers, avoiding the interaction with membrane transporters. Once again, the nanocarrier governs QTP brain penetration with the same rate previously observed $\left(Q_{\text {nano }} 0.067 \mathrm{l} / \mathrm{h}\right.$ per kilogram). However, this rate is now higher than that observed for the unbound QTP brain penetration, which is reduced $\left(\mathrm{CL}_{\mathrm{D}, \mathrm{in}}, \mathrm{SCZ} 0.019 \mathrm{l} / \mathrm{h}\right.$ per kilogram) in SCZ-like animals, resulting in an increase in brain penetration. Comparing the increased brain exposure observed in SCZlike animals when QLNC was administered, it is possible to note that the nanocarriers returned QTP concentration levels to those observed in naive animals.

The nanocarrier used in the present work is composed of a lipid-dispersed core (medium chain triglycerides and sorbitan monostearate) enveloped by a polymeric wall [poly( $\varepsilon$-caprolactone)], leading to a drug release kinetic dependent on both the core viscosity (in vivo represented by $\mathrm{F}_{\text {core }}$ and $\mathrm{K}_{\text {core }}$ ) and the polymeric wall (in vivo represented by $\mathrm{F}_{\text {surface }}$ and $\mathrm{K}_{\text {external }}$ ). Drug release rate from this nanoparticle decreases according to the increase in the polymer concentration (Cruz et al., 2006) or depending on medium chain triglycerides and/or sorbitan monostearate concentrations. An increase in sorbitan monoestearate concentration, for instance, might cause a resistance to drug diffusion from the core of the LNC (Jäger et al., 2009).

The improvement in QTP delivery to the brain observed with QLNC sheds light on the pharmacokinetic component of 
the antipsychotic treatment resistance, opening the possibility for use of nanocarriers to improve SCZ treatment limited response rates.

The innovative strategy described in the present work, combining experimental microdialysis and population pharmacokinetic (popPK) modeling, applied to the investigation of nanocarrier brain penetration in SCZ-like animals provided an understanding of the drug delivery mechanism from the nanostructure in vivo and an opportunity to build a tool that can be used to simulate distinct outcomes aiding in the development of nanocarriers with tailored delivery rates.

A semimechanistic model of total and unbound plasma and unbound brain concentrations allowed for the determination of the rate and extent of QTP brain distribution following FQ and QLNC dosing to naive and SCZ-like rats. The develop model is useful for better understanding the potential of lipid core nanocapsules for drug delivery to the brain, opening the opportunity of using this approach to design nanoformulation for improving SCZ-treatment limited response rates.

\section{Acknowledgments}

The authors acknowledge Dr. Liberato Brum Junior (Prati Donaduzzi, Toledo, PR, Brazil) for kindly providing quetiapine fumarate and technical support from the Animal's Facility staff (UFRGS/ Biochemistry Department).

\section{Authorship Contributions}

Participated in research design: Carreño, Herrmann, Kuze Rates, Dalla Costa.

Conducted experiments: Carreño, Helfer, Staudt, Olivo, Paese, Meyer.

Contributed new reagents or analytic tools: Guterres, Kuze Rates.

Performed data analysis: Carreño, Trocóniz, Dalla Costa.

Wrote or contributed to the writing of the manuscript: Carreño, Trocóniz, Dalla Costa.

\section{References}

Araújo BV, Silva CF, Haas SE, and Dalla Costa T (2008) Microdialysis as a tool to determine free kidney levels of voriconazole in rodents: a model to study the technique feasibility for a moderately lipophilic drug. J Pharm Biomed Anal 47: 876-881.

Boulton DW, DeVane CL, Liston HL, and Markowitz JS (2002) In vitro P-glycoprotein affinity for atypical and conventional antipsychotics. Life Sci 71:163-169.

Carreño F, Helfer VE, Staudt KJ, Paese K, Meyer FS, Herrmann AP, Guterres SS, Rates SMK, and Dalla Costa T (2020) Quetiapine lipid core nanocapsules restore prepulse inhibition deficits in a neurodevelopmental model of schizophrenia in male and female rats. Schizophr Res 218:173-179.

Carreño F, Paese K, Silva CM, Guterres SS, and Dalla Costa T (2015) Characterizing the mechanism of quetiapine distribution in lipid-core nanocapsules pseudo-phases using a validated LC/UV method. Quim Nova 38:1181-1186.

Carreño F, Paese K, Silva CM, Guterres SS, and Dalla Costa T (2016a) Pre-clinical investigation of the modulation of quetiapine plasma pharmacokinetics and tissues biodistribution by lipid-core nanocapsules. J Pharm Biomed Anal 119:152-158.

Carreño F, Paese K, Silva CM, Guterres SS, and Dalla Costa T (2016b) Pharmacokinetic investigation of quetiapine transport across blood-brain barrier mediated by lipid core nanocapsules using brain microdialysis in rats. Mol Pharm 13 $1289-1297$.

Cruz L, Soares LU, Costa TD, Mezzalira G, da Silveira NP, Guterres SS, and Pohlmann AR (2006) Diffusion and mathematical modeling of release profiles from nanocarriers. Int $J$ Pharm 313:198-205.

de Klerk OL, Willemsen AT, Bosker FJ, Bartels AL, Hendrikse NH, den Boer JA, and Dierckx RA (2010) Regional increase in P-glycoprotein function in the blood-brain barrier of patients with chronic schizophrenia: a PET study with [(11) Clverapamil as a probe for P-glycoprotein function. Psychiatry Res 183:151-156.

Dimer FA, Pigatto MC, Boque CA, Pase CS, Roversi K, Pohlmann AR, Burger ME, Rates MK, Dalla Costa T, and Guterres SS (2015) Nanoencapsulation improves relative bioavailability and antipsychotic effect of olanzapine in rats. $J$ Biomed Nanotechnol 11:1482-1493.

Gilkey MJ, Krishnan V, Scheetz L, Jia X, Rajasekaran AK, and Dhurjati PS (2015) Physiologically based pharmacokinetic modeling of fluorescently labeled block copolymer nanoparticles for controlled drug delivery in leukemia therapy. CPT Pharmacometrics Syst Pharmacol 4:e00013.

Han M, Huang XF, Chen DC, Xiu MH, Hui L, Liu H, Kosten TR, and Zhang XY (2012) Gender differences in cognitive function of patients with chronic schizophrenia. Prog Neuropsychopharmacol Biol Psychiatry 39:358-363.

He Q, Liu J, Liang J, Liu X, Li W, Liu Z, Ding Z, and Tuo D (2018) Towards improvements for penetrating the blood-brain barrier-recent progress from a material and pharmaceutical perspective. Cells 7:24-45.

Jäger E, Venturini CG, Poletto FS, Colomé LM, Pohlmann JP, Bernardi A, Battastini AM, Guterres SS, and Pohlmann AR (2009) Sustained release from lipid-core nanocapsules by varying the core viscosity and the particle surface area. J Biomed Nanotechnol 5:130-140.

Jonsson EN and Karlsson MO (1999) Xpose--an S-PLUS based population pharmacokinetic/pharmacodynamic model building aid for NONMEM. Comput Methods Programs Biomed 58:51-64.

Keizer RJ, Karlsson MO, and Hooker A (2013) Modeling and simulation workbench for NONMEM: tutorial on Pirana, PsN, and Xpose. CPT Pharmacometrics Syst Pharmacol 2:e50.

Kennedy JL, Altar CA, Taylor DL, Degtiar I, and Hornberger JC (2014) The social and economic burden of treatment-resistant schizophrenia: a systematic literature review. Int Clin Psychopharmacol 29:63-76.

Li M, Al-Jamal KT, Kostarelos K, and Reineke J (2010) Physiologically based pharmacokinetic modeling of nanoparticles. ACS Nano 4:6303-6317.

Li M, Zou P, Tyner K, and Lee S (2017) Physiologically based pharmacokinetic (PBPK) modeling of pharmaceutical nanoparticles. AAPS J 19:26-42.

Lindbom L, Pihlgren P, and Jonsson EN (2005) PsN-Toolkit--a collection of computer intensive statistical methods for non-linear mixed effect modeling using NONMEM [published correction appears in Comput Methods Programs Biomed (2005) 80 277]. Comput Methods Programs Biomed 79:241-257.

Lindqvist A, Fridén M, and Hammarlund-Udenaes M (2016) Pharmacokinetic considerations of nanodelivery to the brain: using modeling and simulations to predict the outcome of liposomal formulations. Eur J Pharm Sci 92:173-182.

Lindqvist A, Rip J, Gaillard PJ, Björkman S, and Hammarlund-Udenaes M (2013) Enhanced brain delivery of the opioid peptide DAMGO in glutathione pegylated liposomes: a microdialysis study. Mol Pharm 10:1533-1541.

Müller Norbert, Weidinger Elif, Leitner Bianka, and Schwarz Markus J (2015) The role of inflammation in schizophrenia. Front Neurosci 9:372, doi: 10.3389/ fnins.2015.00372 26539073

Paxinos G and Watson C (2014) Paxinos and Watson's The Rat Brain in Stereotaxic Coordinates, Elsevier/Academic Press, Amsterdam.

Pira L, Mongeau R, and Pani L (2004) The atypical antipsychotic quetiapine increases both noradrenaline and dopamine release in the rat prefrontal cortex Eur J Pharmacol 504:61-64.

Pritchett-Corning KR, Chang FT, and Festing MFW (2009) Breeding and housing laboratory rats and mice in the same room does not affect the growth or reproduction of either species. J Am Assoc Lab Anim Sci 48:492-498.

Rodrigues SF, Fiel LA, Shimada AL, Pereira NR, Guterres SS, Pohlmann AR, and Farsky SH (2016) Lipid-core nanocapsules act as a drug shuttle through the blood brain barrier and reduce glioblastoma after intravenous or oral administration. J Biomed Nanotechnol 12:986-1000.

Tunblad K, Hammarlund-Udenaes M, and Jonsson EN (2004) An integrated model for the analysis of pharmacokinetic data from microdialysis experiments. Pharm Res 21:1698-1707.

Wählby U, Jonsson EN, and Karlsson MO (2002) Comparison of stepwise covariate model building strategies in population pharmacokinetic-pharmacodynamic analysis. AAPS PharmSci 4:E27.

Yamamura S, Ohoyama K, Hamaguchi T, Kashimoto K, Nakagawa M, Kanehara S, Suzuki D, Matsumoto T, Motomura E, Shiroyama T, et al. (2009) Effects of quetiapine on monoamine, GABA, and glutamate release in rat prefrontal cortex. Psychopharmacology (Berl) 206:243-258

Yuan D, He H, Wu Y, Fan J, and Cao Y (2019) Physiologically based pharmacokinetic modeling of nanoparticles. J Pharm Sci 108:58-72.

Address correspondence to: Dr. Teresa Dalla Costa, Programa de PósGraduação em Ciências Farmacêuticas, Faculdade de Farmácia, Universidade Federal do Rio Grande do Sul, Av. Ipiranga, 2759, Porto Alegre, Rio Grande do Sul 90610-000, Brazil. E-mail: dalla.costa@ufrgs.br 